countries, with the help of scientific achievements, have done a great deal for the betterment of the everyday life of man. Yet in the West, because of the restlessness, unhappiness, and dissatisfaction of the mind, there are constant conflicts and frustrations resulting in extreme forms of mental disorders, nerve troubles, and functional ailments. This shows that the modern ways of life, based on scientific discoveries and their uses, have not solved the psychological problems of the West. On the other hand, the problems are becoming more and more frightening. So the West must find a way out of this dangerous situation".

D. M. WhEATLEY

\section{QUASI-ABELIAN FUNCTIONS}

\section{Funzioni quasi-abeliane}

By Prof. Francesco Severi. (Pontificiæ Academiæ Scientiarum Scripta Varia 4.) Pp. 327. (Rome: Pontifical Academy of Sciences, 1947.) n.p.

$\mathrm{T}$ HE present treatise, constituting almost entirely an original work, was written during 1944-45 and communicated as a memoir to the Pontifical Academy of Sciences, by which it has been published as one of a series of monographs. Its purpose is to fill an obvious gap both in the theory of algebraic functions and their integrals and in the relevant geometrical literature, subjects to which Prof. Severi has made masterly contributions during the last forty-five years. The concept of quasi-Abelian function, in its simplest form, has its origin in a theorem of Weierstrass, which asserts that the only uniform functions of a complex variable possessing an algebraic addition theorem are the elliptic functions and their degenerate forms, namely, the exponential and rational functions. The functions of the last two types are the quasiAbelian functions relative to the elliptic function.

In his treatise Severi is primarily concerned with the solutions of the generalized problem, that is, the uniform functions of any number of complex variables which possess an algebraic addition theorem; for solutions of the most general kind-the so-called Abelian functions-there already exists a very great literature extending over more than a century, but hitherto there has been no systematic treatment of the degenerate forms. As Severi shows, these are likewise periodic functions in general, though with fewer periods than the associated Abelian functions, and in many respects are strictly analogous to the Abelian functions themselves.

Severi's investigation is, naturally enough, in the tradition of the Italian school of algebraic geometry which, taking its cue from the Germans Brill and Noether, has translated the discoveries of Abel, Jacobi and Riemann concerning multiply-periodic functions into the language of the geometry of curves. For this purpose it employs a strikingly simple technique, depending on the consideration of sets of points on an algebraic curve, from which results not only a remarkable gain in elegance and power, but also a method capable of extension to similar problems of a more complex nature. To this method Severi has now added a concept which has already played an essential part in other researches of his: thus while, according to the classical theory, an algebraic plane curve of degree $n$, possessing $d$ double points, has a genus $p$ given by the formula $p=\frac{1}{8}(n-1)(n-2)-d$, Severi's innovation con. sists in regarding a certain number of these nodes as virtually non-existent, so that the curve has a virtual genus greater than $p$.

The first section of the treatise-about one-third of the whole-is in the nature of a prelude, devoted to the theory of series of points on a curve. The next two sections deal with the quasi-Abelian functions in all their generality, their periods, the associated integrals and allied geometrical results concerning the so-called Picard variety attached to a curve of genus $p$. Severi begins by posing three alternative definitions of the functions, and eventually shows that all three are equivalent to the one already stated above; here, it must be noted, certain parts of the theory hinge on a hypothesis of a geometrical character which has so far not been established. The last section, which is comparatively brief, completes an interesting chapter in the theory of surfaces. It is just forty years since Enriques and Severi wrote their prize memoir (Acta Mathematica, 1909-10) on hyperelliptic surfaces, that is, surfaces the coordinates of the points of which are expressible as uniform quadruply-periodic functions of two independent variables; an example is Kummer's quartic surface, which itself includes Fresnel's wave surface as a special case. The memoir has now been supplemented by a study of the quasi-hyperelliptic surfaces, which comprise Plücker's quartic surface, a familiar locus in line geometry; Severi shows how this is obtainable as a limiting form of Kummer's surface.

The book, which concludes with a detailed bibliography, is a notable addition to the distinguished author's publications, and will doubtless occupy a permanent place in the literature. L. RoTH

\section{BLOOD PRESSURE}

\section{Blood Pressure and its Disorders, including Angina Pectoris}

By Dr. John Plesch. Second edition, revised and enlarged. Pp. xiv $+307+15$ plates. (London : Baillière, Tindall and Cox, 1947.) 21s. net.

$T$ HE first edition of this book by Prof. Plesch will be well known to most workers interested in this subject, and a second edition of it will be welcomed. Most of the chapters have been enlarged and some new ones have been added; references to the literature and case-histories inerease the value of the book, and suggestions made by readers of the first edition have been incorporated. The author says that he has dealt only with aspects of the subject of which he has had practical experience through research and observation, and that he does not intend to present the reader with a text-book. This note of personal experience is evident on every page. It is a book for the progressive and open-minded worker who shares its author's evident desire to understand thoroughly the origins of the diseases discussed and to relieve the suffering that they cause by treatment based upon this understanding.

A short review cannot give an idea of the wide field covered by the text. Planned in three main sections, one on arterial blood pressure, one on venous pressure and one on the much-discussed conception of angina pectoris, the book deals with the physiological basis of disorders of the blood-pressure, their diagnosis and the clinical application of the facts discussed. No doubt many readers will be especially interested in the section entitled "Angina Pectoris", partly because the author does not restrict the conception 